Draft version August 24, 2021

Preprint typeset using IATEX style emulateapj v. 03/07/07

\title{
A GIANT RADIO HALO IN THE MASSIVE AND MERGING CLUSTER ABELL 1351
}

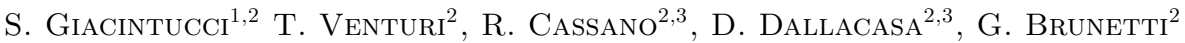 \\ Draft version August 24, 2021
}

\begin{abstract}
We report on the detection of diffuse radio emission in the X-ray luminous and massive galaxy cluster A 1351 ( $\mathrm{z}=0.322)$ using archival Very Large Array data at $1.4 \mathrm{GHz}$. Given its central location, morphology, and Mpc-scale extent, we classify the diffuse source as a giant radio halo. X-ray and weak lensing studies show A 1351 to be a system undergoing a major merger. The halo is associated with the most massive substructure. The presence of this source is explained assuming that mergerdriven turbulence may re-accelerate high-energy particles in the intracluster medium and generate diffuse radio emission on the cluster scale. The position of $\mathrm{A} 1351$ in the $\log \mathrm{P}_{1.4 \mathrm{GHz}}-\log \mathrm{L}_{\mathrm{X}}$ plane is consistent with that of all other radio-halo clusters known to date, supporting a causal connection between the unrelaxed dynamical state of massive $\left(>10^{15} M_{\odot}\right)$ clusters and the presence of giant radio halos.

Subject headings: acceleration of particles, galaxies: clusters: individual (A1351), radiation mechanisms: non-thermal, radio continuum: general - $\mathrm{X}$-rays: general
\end{abstract}

\section{INTRODUCTION}

Beyond the galaxies and the hot gas, clusters of galaxies host non-thermal components in the form of $\mu \mathrm{G}$ magnetic fields and relativistic particles mixed with the thermal intracluster medium (ICM), which prove themselves through steep-spectrum diffuse radio emission in the form of radio halos and relics (see Ferrari et al. (2008) for a recent review). These powerful sources (radio power $\mathrm{P}_{1.4 \mathrm{GHz}} \sim 10^{24}-10^{25} \mathrm{~W} \mathrm{~Hz}^{-1}$ ) are extended on the Mpcscale and are characterized by very low surface brightness $\left(\mu \mathrm{Jy} \operatorname{arcsec}^{-2}\right.$ at $\left.1.4 \mathrm{GHz}\right)$. The origin of these sources has long been considered a puzzle, due to their rare occurrence and to the unclear origin of the radiating relativistic particles (e.g., Brunetti (2008) and Cassano (2009) for recent reviews).

Radio halos are always found in unrelaxed clusters (Buote 2001; Venturi et al. 2008) showing significant substructure in the X-ray images, as well as complex gas temperature distribution (e.g., Govoni et al. 2004; Giacintucci et al. 2005, 2009), which are clear signatures of very recent or ongoing merger events. This suggests a link between the gravitational process of cluster formation and the origin of radio halos. The presence of a correlation between the radio power of halos and the $\mathrm{X}$ ray luminosity, mass, and temperature of the host cluster further supports a tight connection between the thermal and non-thermal components in the ICM (Liang et al. 2000; Govoni et al. 2001; Cassano et al. 2006, hereinafter C06). Recent work based on the Giant Metrewave Radio Telescope (GMRT) radio halo survey (Venturi et al. 2007, 2008; hereinafter V07, V08), carried out with the GMRT at $610 \mathrm{MHz}$, firmly established that (1) radio halos are not ubiquitous in clusters, with only $\sim 30 \%$ of the selected X-ray luminous clusters hosting a radio halo (V08; Cassano et al. 2008, hereinafter C08), and (2) the distribution of radio halos in the $\mathrm{P}_{1.4 \mathrm{GHz}}-$ clus-

\footnotetext{
${ }^{1}$ Harvard-Smithsonian Center for Astrophysics, 60 Garden Street, Cambridge, MA 02138, USA

2 INAF/IRA, via Gobetti 101, I-40129 Bologna, Italy

${ }^{3}$ Dip. Astronomia, University of Bologna, via Ranzani 1, I40127 Bologna, Italy
}

ter X-ray luminosity $\left(\mathrm{L}_{\mathrm{X}}\right)$ plane is bimodal: clusters with and without radio halos are clearly separated, with radio halos always found in dynamically disturbed systems (Brunetti et al. 2007; V08).

A model that explains the origin of radio halos is provided by the so-called turbulent re-acceleration scenario (Brunetti et al. 2001; Petrosian 2001), where it is assumed that magnetohydrodynamic (MHD) turbulence, injected in the ICM during cluster-cluster mergers, has an important role in the re-acceleration of relativistic electrons. According to this scenario, radio halos are expected to be relatively rare and transient phenomena (with a lifetime $\leq 1 \mathrm{Gyr}$ ) generated in dynamically active clusters. The fraction of galaxy clusters with radio halos is expected to increase with cluster mass, since mergers in massive clusters are expected to generate enough turbulence to accelerate relativistic electrons at energies of several GeV (Cassano \& Brunetti 2005).

Based on the above arguments, and motivated by the weak lensing analysis of the massive and unrelaxed cluster A 1351 (Holhjem et al. 2009, hereinafter H09, and references therein), not known to host a radio halo, we searched the Very Large Array (VLA) data archive to check for possible hints of diffuse radio emission on the cluster scale.

In this Letter, we report on the detection of a giant radio halo at the center of A 1351 using archival VLA data at $1.4 \mathrm{GHz}$. The radio observations and data reduction are described in Section 2, the radio images and their analysis are reported in Section 3, results are presented and discussed in Section 4 summary and conclusions are given in Section 5 .

We adopt the $\Lambda$ CDM cosmology with $\mathrm{H}_{0}=70 \mathrm{~km} \mathrm{~s}^{-1}$ $\mathrm{Mpc}^{-1}, \Omega_{m}=0.3$ and $\Omega_{\Lambda}=0.7$. At the redshift of A $1351(z=0.322)$ this leads to a linear scale of $1^{\prime \prime}=4.68$ kpc.

\section{A 1351 AND THE RADIO DATA}

A 1351 is a rich galaxy cluster at redshift $\mathrm{z}=0.322$ with an X-ray luminosity of $\mathrm{L}_{\mathrm{X}}[0.1-2.4] \mathrm{keV}=8.4 \times 10^{44} \mathrm{~h}_{70}{ }^{-2}$ erg $\mathrm{s}^{-1}$ (Allen et al. 2003) and a total mass of 
$M_{200} \simeq 1.17 \pm 0.31 \times 10^{15} h_{70}^{-1} M_{\odot}(\mathrm{H} 09) . \mathrm{X}$-ray images show significant substructure, suggesting an ongoing major merger (Allen et al. 2003). The cluster has a high optical velocity dispersion $\left(\sigma_{\mathrm{v}}>1000 \mathrm{~km} \mathrm{~s}^{-1} ; \mathrm{H} 09\right.$ and references therein), as commonly found in merging systems. The merging scenario is supported by the weak lensing analysis presented in H09: two significant mass concentrations are detected in the weak lensing reconstruction of the cluster mass density, coincident with the galaxy and $\mathrm{X}$-ray gas distribution. The two mass substructures are located at a projected distance of $\sim 3^{\prime}(\sim 840 \mathrm{kpc})$ along the northeast/southwest axis, suggesting that this is the direction of the merger.

A 1351 was observed with the VLA at $1.4 \mathrm{GHz}$ in the A-, D- and C-array configurations in 1994 April, 1995 March and 2000 April, respectively. The details on these observations are summarized in Table 1 , where the columns provide the following information: VLA project, frequency, total bandwidth, array configuration, total time on source, observing date, half-power bandwidth $(\mathrm{HPBW})$, and rms level $(1 \sigma)$ in the full resolution image from each individual data set.

The data were calibrated and reduced using the Astronomical Image Processing System (AIPS), following the standard procedure. Each data set was self-calibrated in phase to correct for residual phase variations. Both uniform and natural weighting were used to produce the final images for each array configuration. The achieved sensitivity $(1 \sigma)$ ranges from 50 to $200 \mu \mathrm{Jy} \mathrm{b}^{-1}$ (see Table 11 , the spread being mostly due to the different observing time. The final self-calibrated data sets were then combined together in a single data set providing a UV range of $0.2-175 \mathrm{k} \lambda$. The shortest baselines ensure the proper imaging of extended radio emission up to $\sim 7^{\prime} .5$ in angular size, corresponding to $\sim 2.1 \mathrm{Mpc}$ at the redshift of A 1351. The high angular resolution provided by the longest baselines allows us to identify and separate possible discrete radio sources projected onto the diffuse emission. With the final combined array we made a set of images, with resolutions ranging from $6^{\prime \prime} .7 \times 5^{\prime \prime} .5$ (full resolution, uniform weighting and $1 \sigma=30 \mu \mathrm{Jy} \mathrm{b}^{-1}$ ) to $20^{\prime \prime} .2 \times 17^{\prime \prime} .8$ (natural weighting, taper, and $1 \sigma=60 \mu \mathrm{Jy}$ $\left.\mathrm{b}^{-1}\right)$. The average residual amplitude errors in the data are on the order of $\lesssim 5 \%$.

\section{THE RADIO IMAGES}

The left panel of Figure 1 shows the $1.4 \mathrm{GHz}$ image of the radio emission from the cluster, at the resolution of $16^{\prime \prime} .2 \times 13^{\prime \prime} .6$, obtained from the combination of the C- and D-array data sets. Diffuse emission is clearly present beyond the individual sources. The right panel of Figure 1 shows a $6^{\prime \prime} .7 \times 5^{\prime \prime} .5$ image obtained with the combined arrays, overlaid on the Sloan Digital Sky Survey (SDSS) red optical image, to highlight the optically identified radio sources (labeled from A to $G$ ) detected in the cluster region. Source A is extended and is associated with SDSS J114224.77+583205.3, the brightest cluster galaxy (BCG). The diffuse emission has no obvious optical counterpart, and permeates the cluster region south of the BCG.

Figure 2 presents a low-resolution image of the radio emission at the cluster center, overlaid on the smoothed X-ray image obtained from archival ROSAT High Reso- lution Imager (HRI) data. The radio image was produced after subtraction of the discrete radio galaxies (A to $\mathrm{G}$ in Figure 1), and using the UV range $0.2-16 \mathrm{k} \lambda$. The image confirms the presence of a diffuse radio source extending on the cluster scale, whose elongation and overall size are in good agreement with the brightest region detected in the X-ray. The radio morphology is irregular, with a peak of emission coincident with the X-ray peak. A southern feature, which we label ridge, is spatially coincident with an edge in the X-ray image. In the northern region a faint filament of radio emission extends toward the northeast, similar to the X-ray emission. Given its location, morphology, and largest linear size of $\sim 1.1 \mathrm{Mpc}$, we classify the diffuse source in A 1351 as a giant radio halo. The ridge is the brightest and most peripheral region of the halo, and its location with respect to the $\mathrm{X}$-ray brightness distribution raises the possibility that it might be a radio relic projected onto the radio halo emission.

Comparison between the morphology of the radio halo (Figure 2) and the mass reconstruction in H09 (their Figure 5) shows that the halo is associated with the most massive cluster substructure, and the northern radio filament (Figure 2 points toward a secondary peak both in the mass reconstruction and number density of galaxies. The total flux density of the radio halo in Figure 2 is $\mathrm{S}_{1.4 \mathrm{GHz}}=32.4 \mathrm{mJy}$, corresponding to a radio power $\mathrm{P}_{1.4 \mathrm{GHz}}=1.17 \times 10^{25} \mathrm{~W} \mathrm{~Hz}{ }^{-1}$. The ridge accounts for approximately $40 \%$ of the total flux density $\left(\mathrm{P}_{1.4 \mathrm{GHz}}=\right.$ $\left.4.67 \times 10^{24} \mathrm{~W} \mathrm{~Hz}^{-1}\right)$. We note that the contribution of residual emission from the head-tail (source A in Figure 1) is $\lesssim 4 \mathrm{mJy}$, as estimated from the comparison of the Hux measurements on a set of different resolution images of the whole radio emission from the cluster (including the diffuse emission and the contribution of the individual radio galaxies).

\section{DISCUSSION}

The current picture of radio halo formation in galaxy clusters is very complex and tightly connected to the process of formation and evolution of the host cluster (e.g., Brunetti (2008) for a recent review). Monte Carlo simulations, carried out under the hypothesis that MHD turbulence in cluster mergers may re-accelerate highenergy particles, show that about $30 \%$ of X-ray luminous $\left(\mathrm{L}_{\mathrm{X}}>8 \times 10^{44} \mathrm{erg} \mathrm{s}^{-1}\right.$ ) galaxy clusters at $\mathrm{Z} \sim 0.2$ 0.3 are expected to host a giant radio halo (C06; C08). In this context, the presence of diffuse cluster-scale radio emission in A 1351 is not surprising since the cluster is a massive and $\mathrm{X}$-ray luminous system experiencing a major merger event (Allen et al. 2003; H09). In particular, Cassano \& Brunetti (2005) derived an analytical formula for the turbulent-acceleration rate based on a single-merger scenario. Assuming a redshift $\mathrm{z}=0.322$, we find that steep-spectrum Mpc-scale radio emission at 1.4 $\mathrm{GHz}$ (that requires acceleration timescales $\leq 0.3 \mathrm{Gyr}$ ) is expected to be generated in connection with a merger with mass ratio $\leq 2.8$, for a total virial mass of the system $M_{v} \simeq 1.2 \times 10^{15} M_{\odot}$ (consistent with the weak lensing analysis in $\mathrm{H} 09$ ), or $\leq 4$, for $M_{v} \simeq 2 \times 10^{15} M_{\odot}$ (derived from the $L_{x}-M_{v}$ correlation; e.g., C06). Furthermore, based on the GMRT observations in V07 and V08 and literature data, C08 showed that the fraction of clusters with radio halo in the redshift range $0.20-0.32$ 
TABLE 1

Details of the VLA archival observations

\begin{tabular}{cccccccc}
\hline $\begin{array}{c}\text { VLA } \\
\text { Project }\end{array}$ & $\begin{array}{c}\text { Frequency } \\
(\mathrm{GHz})\end{array}$ & $\begin{array}{c}\Delta \nu \\
(\mathrm{MHz})\end{array}$ & Config. & $\begin{array}{c}\text { Obs. Time } \\
(\text { minutes })\end{array}$ & Date & $\begin{array}{c}\text { Beam, P.A. } \\
\left({ }^{\prime \prime} \times{ }^{\prime \prime},{ }^{\circ}\right)\end{array}$ & $\begin{array}{c}\text { rms } \\
\left(\mu \mathrm{Jy} \mathrm{b} \mathrm{b}^{-1}\right)\end{array}$ \\
\hline AB699 & 1.4 & 25 & $\mathrm{~A}$ & 30 & 1994 Apr & $1.5 \times 1.1,-5$ & 65 \\
$\mathrm{AO} 149$ & 1.4 & 25 & $\mathrm{C}$ & 120 & $2000 \mathrm{Apr}$ & $15.9 \times 13.3,28$ & 50 \\
$\mathrm{AM} 469$ & 1.4 & 25 & $\mathrm{D}$ & 60 & 1995 Mar & $57.9 \times 43.0,50$ & 200 \\
\hline
\end{tabular}
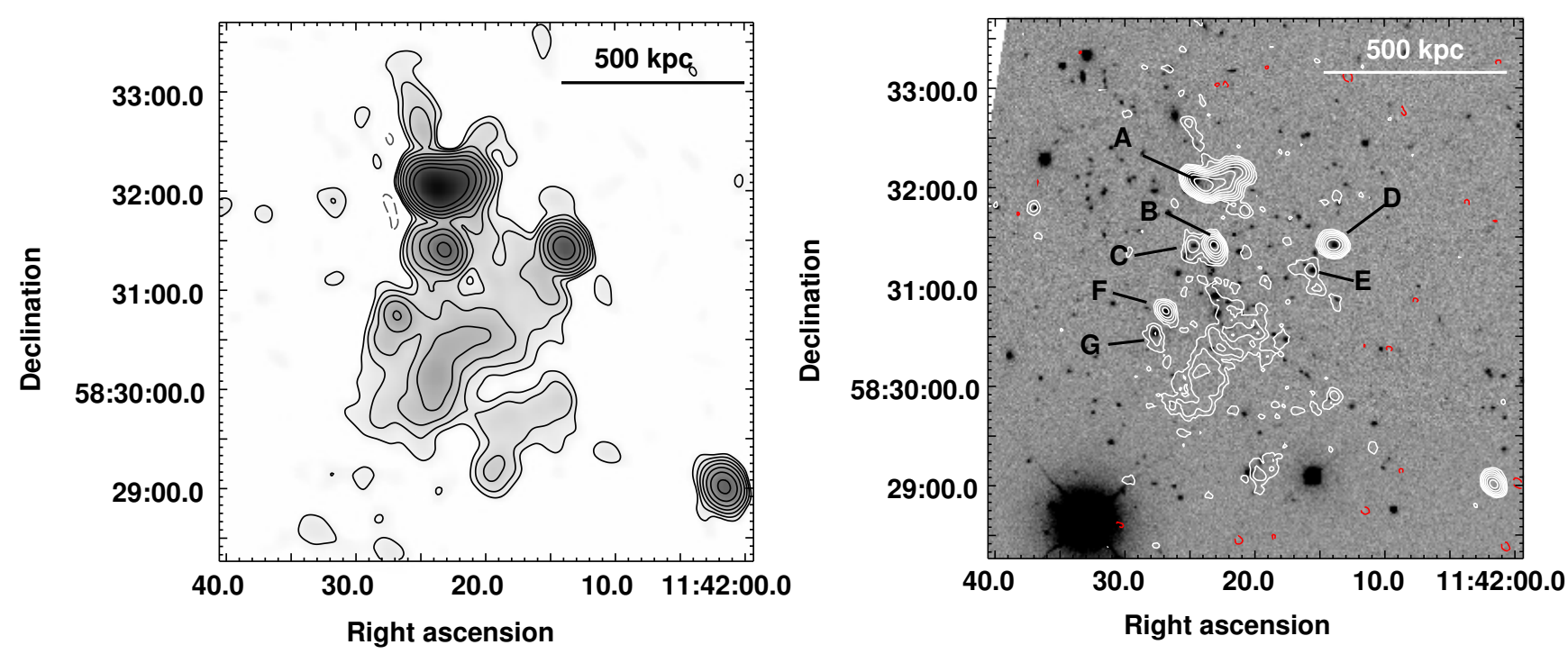

FIG. 1. - Left: VLA $1.4 \mathrm{GHz}$ image (contours and grayscale) obtained from the combination of the C- and D-array data sets. The HPBW is $16^{\prime \prime} .2 \times 13^{\prime \prime} .6$, P.A. $28^{\circ}$. Contours are spaced by a factor 2 from $\pm 3 \sigma=0.15 \mathrm{mJy} \mathrm{b}^{-1}$. Right: VLA 1.4 GHz contours from the combined $(\mathrm{A}+\mathrm{C}+\mathrm{D})$-array data set, superposed to the red optical image from SDSS. The HPBW is $6^{\prime \prime} .7 \times 5^{\prime \prime} .5, \mathrm{P} . \mathrm{A} .40^{\circ}$. Contours are spaced by a factor 2 from $\pm 3 \sigma=0.09 \mathrm{mJy} \mathrm{b}^{-1}$ (negative contours are shown in red). Individual radio galaxies are labeled from A to $\mathrm{G}$.

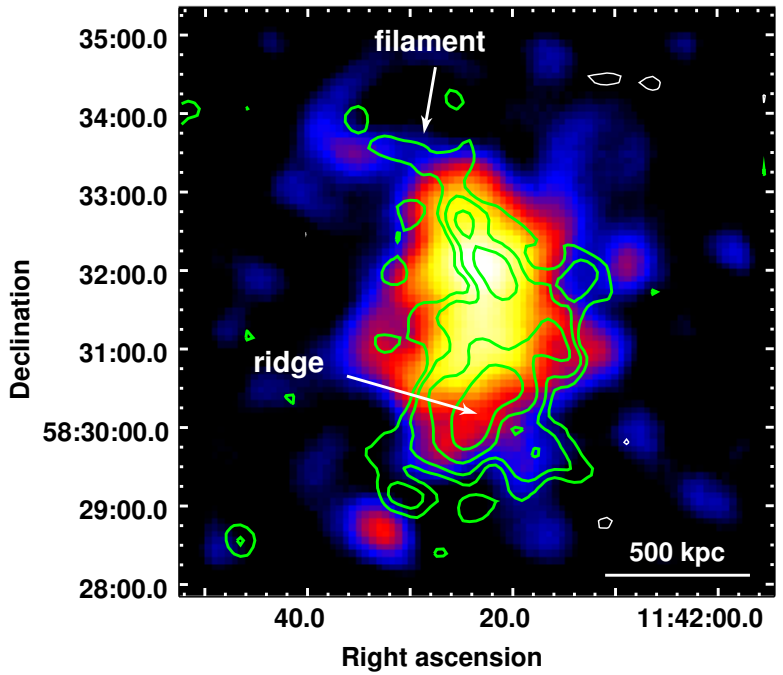

Fig. 2.- VLA $1.4 \mathrm{GHz}$ contours of the extended emission in A 1351, after subtraction of the individual sources (A to G in Figure 1 left panel) from the combined $(\mathrm{A}+\mathrm{C}+\mathrm{D})$-array data set. The image has been obtained using the UV range $0.2-16 \mathrm{k} \lambda$. The HPBW is $20^{\prime \prime} .2 \times 17^{\prime \prime} .8$, P.A. $28^{\circ}$. Contours are spaced by a factor 2 , starting from $\pm 3 \sigma=0.18 \mathrm{mJy} \mathrm{b}^{-1}$ (negative contours are shown in white). The color image is the smoothed ROSAT HRI image of the cluster. and with $L_{X}>8 \times 10^{44} \mathrm{erg} \mathrm{s}^{-1}$ is on the order of $40 \%$, in line with the expectations of the re-acceleration scenario. Remarkably, the X-ray luminosity $\left(L_{X} \simeq 8.4 \times 10^{44} \mathrm{erg}\right.$ $\left.\mathrm{s}^{-1}\right)$ and redshift $(\mathrm{z}=0.32)$ of $\mathrm{A} 1351$ are within these same ranges.

In Figure 3 we show the distribution of the clusters of the GMRT radio halo survey and of other radio-halo clusters from the literature in the $P_{1.4 G H z}-L_{X}$ plane (adapted from Brunetti et al. 2009). The clusters hosting a radio halo are clearly separated from those without. The analysis of the dynamical properties (where available) suggests that such separation is most likely due to the different dynamical state of radio-loud and radioquiet clusters: radio halos are always found in dynamically disturbed clusters, while radio-quiet clusters tend to be relaxed systems (V08). This can be interpreted in the framework of the re-acceleration scenario since clusters are expected to move with time from the radio-quiet region (upper limits in Figure 3) to the $\mathrm{P}_{1.4 G H z}-L_{X}$ correlation in a relatively short timescale (on the order of $\approx 10^{8}$ years, i.e., the re-acceleration timescale of the emitting electrons), leaving the region between radiohalo and radio-quiet clusters poorly populated (Brunetti et al. 2007, 2009).

In Figure 3 we report the position of A 1351 as a magenta symbol. The diffuse radio emission in A 1351 has a complex morphology, and the data currently available 


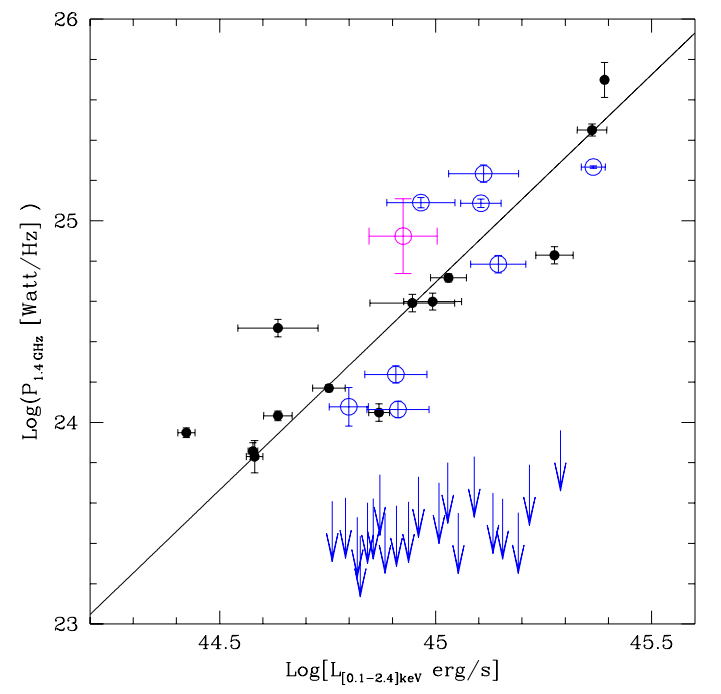

FIG. 3.- Distribution of the GMRT radio-halo clusters (open blue symbols) and other radio-halo clusters from the literature (filled black symbols) in the $P_{1.4 G H z}-L_{X}$ plane (adapted from Brunetti et al. 2009). The magenta point marks the position of A 1351.

do not allow us to establish if the ridge (Figure 2 is part of the radio halo emission or a radio relic located in the southern region of the cluster. For this reason, the $\log \mathrm{P}_{1.4 \mathrm{GHz}}$ value adopted in Figure $3\left(\log \mathrm{P}_{1.4 \mathrm{GHz}}[\mathrm{W}\right.$ $\left.\left.\mathrm{Hz}^{-1}\right]=24.97 \pm 0.19\right)$ is an intermediate value, whose associated large errors account for the possibility that the ridge is either part of the radio halo or unrelated to it. The location of A 1351 is consistent with the distribution of all radio halos known so far. We underline that the plot in Figure 3 includes all the information available in the literature so far: only $\sim 20$ clusters are known to host a giant radio halo, therefore the detection of a new one in a well-constrained merging system contributes to settle the radio-halo cluster merger connection.

\section{CONCLUSIONS}

Using archival VLA data at $1.4 \mathrm{GHz}$ we found a giant radio halo in the massive galaxy cluster $\mathrm{A} 1351$, with a total extent of $\sim 1.1 \mathrm{Mpc}$ and a radio power $\mathrm{P}_{1.4 \mathrm{GHz}}=$ $1.2 \times 10^{25} \mathrm{~W} \mathrm{~Hz}^{-1}$. The host cluster is experiencing a major merger process which is responsible for the complex distribution of the X-ray emitting gas and total mass of the system, as revealed by the X-ray and weak lensing studies. The ongoing merger may generate turbulence in the cluster volume, which in turn may re-accelerate relativistic electrons in the ICM and generate Mpc-scale radio emission. Monte Carlo simulations, carried out under this hypothesis, show that about $30 \%$ of X-ray luminous $\left(\mathrm{L}_{\mathrm{X}}>8 \times 10^{44} \mathrm{erg} \mathrm{s}^{-1}\right)$ galaxy clusters at $\mathrm{z} \sim 0.2-0.3$ are expected to host a giant radio halo (C06, C08). Remarkably, the position of $\mathrm{A} 1351$ in the plane $\mathrm{P}_{1.4 \mathrm{GHz}}-L_{\mathrm{X}}$ is consistent with that of all the other radio-halo clusters known to date, suggesting a causal link between the dynamical state of massive $\left(>10^{15} M_{\odot}\right)$ clusters and the presence of giant radio halos.

Follow-up observations at lower (and multi) frequency will be important to derive the total radio spectrum of the diffuse emission, which is an essential information to discriminate between the theoretical models for the formation of radio halos (e.g., Brunetti et al. 2008, Dallacasa et al. 2009). A spatially resolved image of the spectral index distribution in the halo will also be a useful tool to shed light on the origin of the diffuse radio component in A 1351.

\section{ACKNOWLEDGEMENTS}

This work is partially supported by INAF under grants PRIN-INAF2007 and PRIN-INAF2008 and by ASI-INAF under grant I/088/06/0.

\section{REFERENCES}

Allen, S. W., Schmidt, R. W., Fabian, A. C., \& Ebeling, H., 2003, MNRAS, 342, 287

Brunetti, G., 2008, in Magnetic Fields in the Universe II. Rev. Mex. Astron. Astrof. (SC), eds. A. Esquivel et al., arXiv:0810.0692

Brunetti G.. Cassano R., Dolag K., \& Setti G., Aं\&A, in press (arXiv:0909.2343)

Brunetti, G., Venturi, T., Dallacasa, D., Cassano, R., Dolag, K., Giacintucci, S. \& Setti, G., 2007, ApJ, 670, 5

Brunetti, G., et al. 2008, Nature, 455, 944

Buote, D.A., 2001, ApJ, 553, L15

Cassano, R., 2009, in ASP Conf. Ser., 407, The Low Frequency Radio Universe, ed. D.J. Saikia, D. Green, Y. Gupta, \& T. Venturi (San Francisco, CA:ASP), in press (arXiv:0902.2971) Cassano R. \& Brunetti G., 2005, MNRAS 357, 1313 Cassano R., Brunetti G. \& Setti G., 2006, MNRAS, 369, 1577 (C06) Cassano, R., Brunetti, G., Venturi, T., Setti, G., Dallacasa, D., Giacintucci, S., \& Bardelli, S., 2008, A\&A, 480, 687 (C08)

Dallacasa, D., et al. 2009, ApJ, 699, 1288
Ferrari, C., Govoni, F., Schindler, S., Bykov, A.M., \& Rephaeli, Y., 2008, Space Sci. Rev., 134, 93

Giacintucci, S., et al. 2005, A\&A, 440, 867

Giacintucci S., et al. 2009, A\&A, in press (arXiv:0905.3479)

Govoni, F., Ensslin, T.A., Feretti, L., \& Giovannini G., 2U01, A\&A, 369,441

Govoni, F., Markevitch, M., Vikhlinin, A., Van Speybroek, L., Feretti, L., \& Giovannini, G., 2004, ApJ, 605, 695

Holhjem, K., Schirmer, M., \& Dahle, H., 2009, A\&A, 504, 1 (H09) Liang H., Hunstead R.W., Birkinshaw M., \& Andreani P., 2000, ApJ 544, 686

Petrosian, V., 2001, ApJ 557, 560

Venturi, T., Giacintucci, S., Brunetti, G., Cassano, R., Bardelli, S., Dallacasa, D., \& Setti, G., 2007, A\&A 463, 937 (V07)

Venturi, T., Giacintucci, S., Dallacasa, D., Cassano, R., Brunetti, G., Badelli, S., \& Setti, G., 2008, A\&A, 484, 327 (V08) 